\title{
Gaia Basic Angle Monitoring system
}

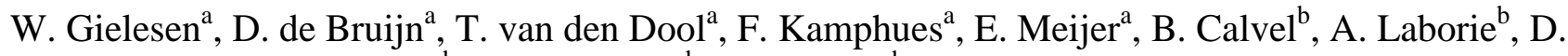

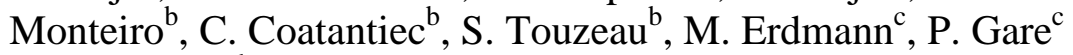 \\ ${ }^{\mathrm{a}} \mathrm{TNO}$ (Delft, the Netherlands); ${ }^{\mathrm{b}}$ EADS Astrium (Toulouse, France); ${ }^{\mathrm{c}}$ ESA ESTEC (Noordwijk, the \\ Netherlands)
}

\begin{abstract}
The Gaia mission ${ }^{1}$ will create an extraordinarily precise three-dimensional map of more than one billion stars in our Galaxy. The Gaia spacecraft ${ }^{2}$, built by EADS Astrium, is part of ESA's Cosmic Vision programme and scheduled for launch in 2013. Gaia measures the position, distance and motion of stars with an accuracy of 24 micro-arcsec using two telescopes at a fixed mutual angle of $106.5^{\circ}$, named the 'Basic Angle'. This accuracy requires ultra-high stability, which can only be achieved by using Silicon Carbide for both the optical bench and the telescopes. TNO has developed, built and space qualified the Silicon carbide Basic Angle Monitoring (BAM) on-board metrology system ${ }^{3}$ for this mission. The BAM measures the relative motion of Gaia's telescopes with accuracies in the range of 0.5 micro-arcsec. This is achieved by a system of two laser interferometers able to measure Optical Path Differences (OPD) as small as 1.5 picometer rms. Following a general introduction to the Gaia mission, the Payload Module (PLM) and the use of Silicon Carbide as base material, this presentation will address an overview of the challenges towards the key requirements, design, integration and testing (including space-level qualification) of the Gaia BAM.
\end{abstract}

Keywords: Gaia, Opto-mechanics, picometer, metrology, stability, Silicon Carbide, cryogenic, space qualification

\section{INTRODUCTION}

\subsection{Gaia mission}

Gaia $^{4}$ is a global space astrometry mission, and a successor to the ESA Hipparcos mission, launched in 1989. Part of ESA's Cosmic Vision program, the Gaia spacecraft (see Figure 1) is being built by EADS Astrium France and is scheduled for launch with a Soyuz launcher from Kourou in 2013. At a distance of 1.5 million kilometres from Earth at Lagrangian point L2, slowly spinning around its axis, Gaia will monitor each target star about 100 times over a five-year period, precisely measuring its distance, movement, and change in brightness. Through comprehensive spectrophotometric classification, it will provide the detailed physical properties of each star observed: characterizing their luminosity, temperature, gravity, and elemental composition. This massive stellar census, will provide the basic observational data to tackle an enormous range of important questions related to the origin, structure, and evolutionary history of our Galaxy.

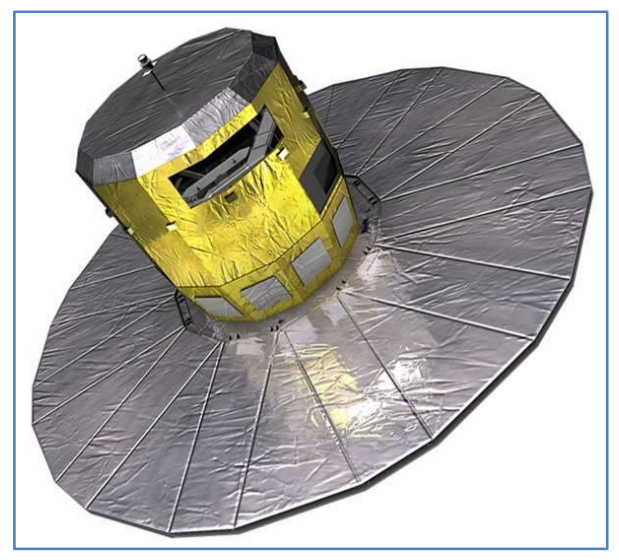

Figure 1: Artists impression of the Gaia satellite (credits: ESA) 
The astronomical measurements performed with Gaia will be accurate to 24 microarcsec, about 100 times more accurate than its predecessor Hipparcos, and comparable to measuring the diameter of a human hair at a distance of 1000 kilometres. To achieve this extreme accuracy at a minimum operational temperature of 100K, the entire Gaia Payload Module is made out of Silicon Carbide (SiC); the first time ever in space history.

\subsection{Gaia Payload Module}

Figure 2 shows the Gaia Payload Module (PLM) consisting of two telescopes with a focal length of $35 \mathrm{~m}$ that re-image the stars on a common focal plane by means of a beam combiner. The two large M1 telescope mirrors (1.45 $\mathrm{m} \mathrm{x} 0.5 \mathrm{~m})$ are fixed on the torus at a mutual angle of $106.5^{\circ}$, named the 'Basic Angle'. The payload structure and mirrors are made entirely out of Silicon Carbide ( $\mathrm{SiC}$ ), for reasons of dimensional stability outlined in section 1.1 above. Due to its design, the overall payload is a-thermal. Remaining small Line Of Sight (LOS) fluctuations of maximum 7 micro-arcsec can only result from thermal gradient fluctuations within the payload. Without compensating for these small LOS fluctuations, the targeted accuracy of Gaia will not be met. Therefore Gaia is equipped with a metrology system that monitors the Basic Angle continuously and enables the correction of the fluctuations by calculation: the Basic Angle Monitoring (BAM) system.
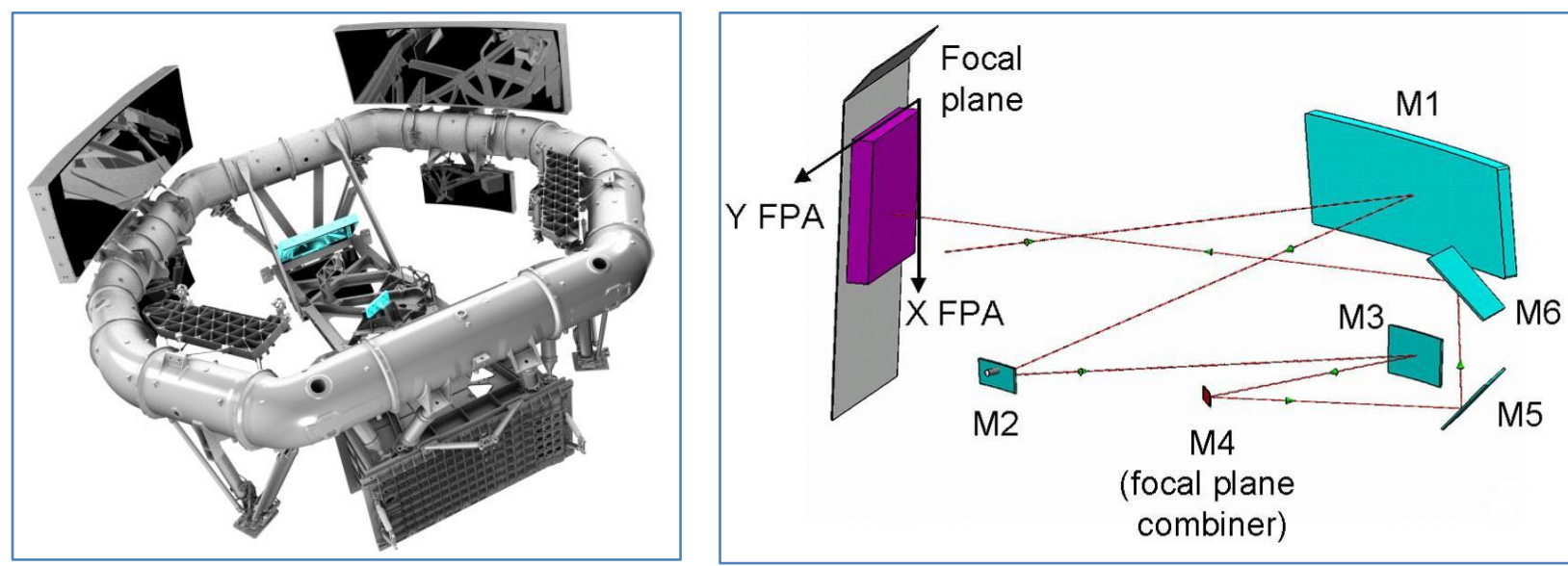

Figure 2: The Gaia Payload Module (PLM) with the two large M1 telescope mirrors on top (left; credits: ESA) and a schematic of the optical configuration (right; credits: EADS ASTRIUM)

\subsection{The Basic Angle Monitoring (BAM) system}

The BAM measures the Basic Angle in flight with an accuracy of about 0.5 micro-arc second rms at 5 minutes intervals of scientific operation. Considering a telescope base length of $0.6 \mathrm{~m}$, this variation corresponds to an optical path difference (OPD) of 1.5 pico-meter rms.
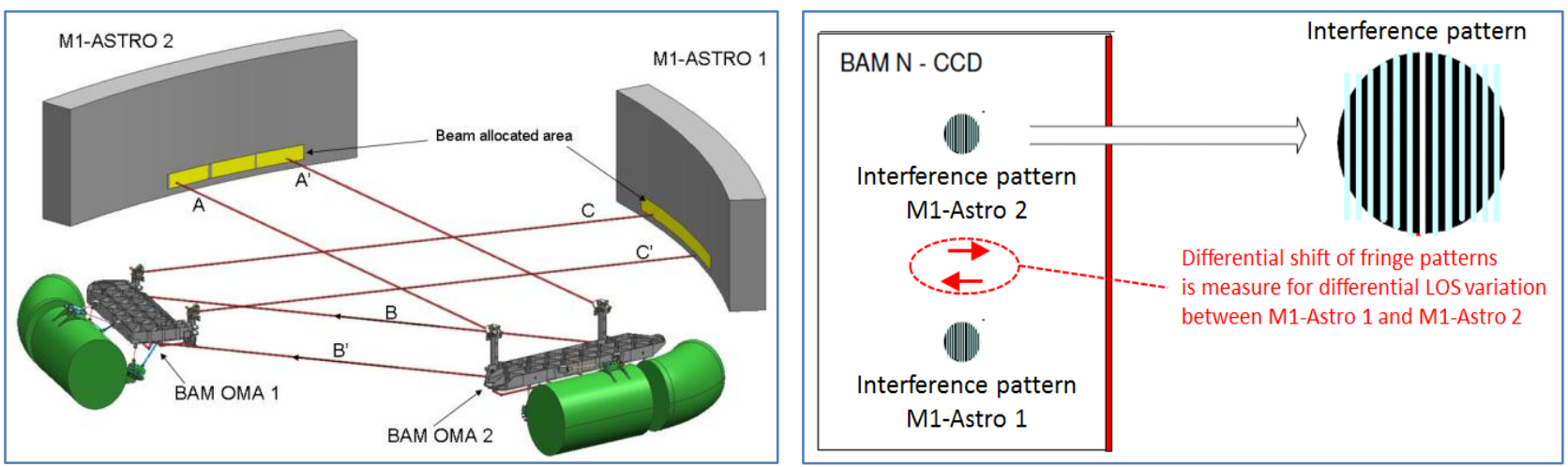

Figure 3: Schematic of Basic Angle Monitoring system with optical beams (left) and principle of fringe patterns on CCD (right). 
The BAM principle, shown in Figure 3, is based on the measurement of the relative position of two interferometric patterns, each one being generated from a common laser diode source. The common beam is split by optics into two pairs that are sent towards the two M1 telescopes Astro 1 and Astro 2 via two 'bars' positioned opposite the M1 mirrors. Both beam pairs are projected on the same CCD in the focal plane of the telescope mirrors. This results in two interference patterns on the BAM CCD. Rotation of a telescope mirror induces differential fringe motion. This provides information about the differential variation of the line-of-sight of each telescope. Therefore the basic angle variations are linked to the differential variation of both Lines Of Sight. Two CCD detectors in the focal plane, a nominal (N) and a redundant $(\mathrm{R}) \mathrm{CCD}$, are dedicated to the BAM function. Each of the two BAM CCD receives the two fringe patterns generated by the corresponding laser source through the two bars.

The BAM bars (BAM OMA 1 and BAM OMA 2) are open-structured base plates supporting optics to create and direct the optical beams (see Figure 4). The light source entrance fibres, collimating optics, filters and beam splitters are only present on BAM OMA bar 2. The base plates, periscopes, folding mirrors, and collimator optics are all made of Sintered Silicon Carbide (SSiC). Some components (transmission optics) are necessarily made of glass; these are the beam splitters (fused silica), attenuator filters (BG40) and polarizers (polarcor ${ }^{\mathrm{TM}}$ ). Each bar is mounted via INVAR iso-static mounts on the Gaia payload main SSiC structure (torus).
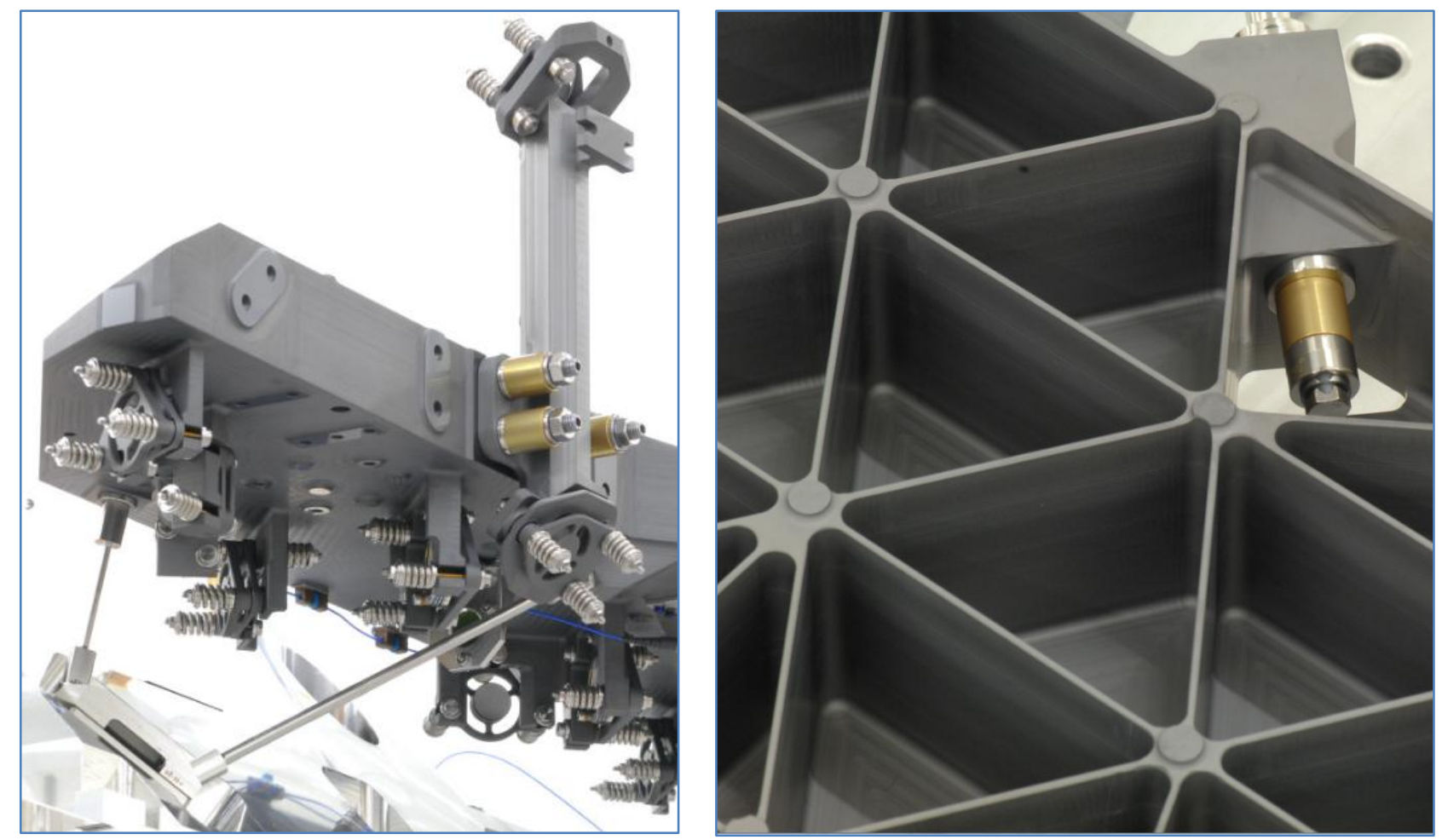

Figure 4: Details of SSiC BAM bar 2 with optics, periscope and Invar iso-static mount (left) and open backside structure of SSiC baseplate (right). Credits: TNO/Fred Kamphues

\subsection{Sintered Silicon Carbide (SSiC)}

To achieve the level of high accuracy and extreme stability at cryogenic temperatures, required for the Gaia mission, the use of exotic materials like Sintered Silicon Carbide (SSiC) or Beryllium has some major benefits. Unlike Beryllium, $\mathrm{SSiC}$ has no toxicity issues during manufacturing and machining and is therefore preferred. The SSiC material used for the Gaia mission was supplied by Boostec, nowadays part of the Mersen group.

$\mathrm{SSiC}$ is a relative lightweight material $\left(\rho=3160 \mathrm{~kg} / \mathrm{m}^{3}\right)$ with a high stiffness $(\mathrm{E}=420 \mathrm{GPa})$ resulting in a very high specific stiffness $\left(E / \rho=135 \times 10^{6} \mathrm{Nm} / \mathrm{kg}\right)$, about 5 times the value of the usual materials used in space: Aluminum, 
Titanium and Steel. As a result, structures of SSiC can be made 5 times lighter to achieve the same stiffness. This is an important criterion for applications where every kilogram is expensive (due to launch costs).

Another advantage, especially of interest to Gaia, is the dimensional stability of SSiC due to the combination of low thermal expansion $(\alpha=2 \cdot 10-6 / \mathrm{K}$; low deformation), a relative high thermal conductivity $(\lambda=180 \mathrm{~W} / \mathrm{m} / \mathrm{K}$; low thermal gradients) and a high degree of isotropy (homogeneous in all directions).

Moreover SSiC is a chemically stable material and can be polished to optical qualities which makes it a suitable material for manufacturing mirrors.

Table 1: Characteristic properties of SSiC compared with other materials. The colour scale clearly shows the benefits of Beryllium and SSiC for these specific properties of interest.

\begin{tabular}{|c|c|c|c|c|c|}
\hline Material & $\begin{array}{l}\text { Aluminum } \\
\text { (6061 T6) }\end{array}$ & $\begin{array}{l}\text { Stainless } \\
\text { Steel } 302\end{array}$ & $\begin{array}{l}\text { Titanium } \\
\text { (Ti6Al4V) }\end{array}$ & Beryllium & $\begin{array}{l}\text { Sintered } \\
\quad \mathrm{SiC}\end{array}$ \\
\hline $\begin{array}{l}\text { Density } \\
\rho\left(\mathrm{kg} / \mathrm{m}^{3}\right)\end{array}$ & 2700 & 7900 & 4430 & 1800 & 3160 \\
\hline $\begin{array}{l}\text { Stiffness } \\
E(G P a)\end{array}$ & 69 & 193 & 114 & 303 & 420 \\
\hline $\begin{array}{l}\text { Specific stiffness } \\
E / \rho\left(\times 10^{6} \mathrm{Nm} / \mathrm{kg}\right)\end{array}$ & 25,5 & 24 & 25,7 & 168 & 135,5 \\
\hline $\begin{array}{l}\text { Therm. conductivity } \\
\lambda(\mathrm{ppm}) @ 20^{\circ} \mathrm{C}\end{array}$ & 167 & 16 & 6,7 & 216 & 180 \\
\hline $\begin{array}{l}\text { Therm. expansion } \\
\alpha(\mathrm{ppm}) @ 20^{\circ} \mathrm{C}\end{array}$ & 23,6 & 17 & 8,6 & 11,4 & 2 \\
\hline
\end{tabular}

Obviously, the use of SSiC for manufacturing complex opto-mechanical instruments also has some drawbacks. The material in its final state (after sintering) is highly brittle and extremely hard. This significantly limits the opportunity for machining the material; the available methods are expensive and time consuming (and most of the time still experimental). Therefore, handling and applying $\mathrm{SSiC}$ parts requires special attention to prevent fracture due to mechanical impacts or stress concentrations.

The properties of SSiC have a major impact on its application both in design solutions, manufacturing, handling and operation.

\section{GAIA BAM PERFORMANCE REQUIREMENTS}

The main performance requirements of the BAM system are derived from the following functional needs:

- The BAM shall generate two beam sets that, through reflection by the Gaia telescope system, shall be projected in the focal plane of the BAM CCD; this applies for both telescopes ASTRO-1 and ASTRO-2 and for both the nominal light path and the redundant light path.

- The beams of each set shall have sufficient overlap in order to create a fringe pattern with sufficient resolution; the white light fringe (or zero path difference fringe) shall be included in each fringe pattern

- $\quad$ The fringe patterns created by the beams shall have sufficient contrast

Table 2 gives an overview of the resulting opto-mechanical requirements applicable at BAM subsystem level. These requirements need to be achieved after alignment and remain within allowable positive margins after qualification testing and unit delivery, storage, satellite integration and transport, launch (vibration), PLM bi-pod release (shock) and at L2 conditions (zero g, vacuum, 120K, radiation) for a minimum in-orbit lifetime of 5,5 years. 
Table 2: Main performance requirements derived for the BAM system.

\begin{tabular}{|l|l|}
\hline \multicolumn{2}{|l|}{ BAM system performance requirements: } \\
\hline Baseline angle (= angle beam sets A $-\mathrm{C}$ ): & $106,5^{\circ} \pm 50 \mu \mathrm{rad}$ \\
\hline Base length difference (= distance A - A' and C - C'): & $600 \pm 60 \mathrm{~mm} ; \pm 1 \mathrm{~mm}$ between beam sets A and C \\
\hline Beam pointing (deviation from nominal angle): & $<100 \mu \mathrm{rad}$ \\
\hline Differential beam tilt (angle between A and A' or C and C'): & $<50 \mu \mathrm{rad}$ \\
\hline OPD (path diff. between A and A' or C and C'): & $<8.5 \mu \mathrm{m}$ \\
\hline WFE (wave front quality of beams): & $25 \mathrm{~nm} \mathrm{rms}$ \\
\hline Transmission (optical throughput of beams): & $>0.15$ \\
\hline
\end{tabular}

Flanked by other requirements and budget limitations that need to be satisfied such as mass, envelope and thermal behaviour it will be obvious that the design, development and realization of the BAM system is more than challenging.

\section{GAIA BAM DESIGN AND DEVELOPMENT CHALLENGES}

Designing a complex opto-mechanical space interferometer that is mainly built of Silicon Carbide requires an entirely different approach than what is custom for e.g. Aluminum instruments.

One of the specific features to be taken into account, particularly for the SSiC type of silicon carbide, is the shrinkage in the order of $16 \%$ that occurs after the sintering at high temperature. Normally, the machining of SiC products is performed in the so-called green phase, prior to sintering. In case the manufacturing drawings are properly scaled to guarantee that the final product after sintering has the correct dimensions, this $16 \%$ shrinkage is not of special concern. However, the variation in shrinking between separate sintering batches can amount up to $0.4 \%$. This can be a major concern in a case as for Gaia BAM, where the larger structures like baseplates cannot be manufactured in the same sintering batch and where interchange-ability of spare baseplates from different batches needs to be guaranteed. On a length of a BAM baseplate of typical $1 \mathrm{~m}$, a variation of $0.4 \%$ results in a potential difference of $4 \mathrm{~mm}$; for a system that needs to be aligned at micro-meter level this is unacceptable and necessitates additional tuning opportunities. More tuning requires more optical components and as a result higher mass, lower opto-mechanical budgets (tilt stability, WFE, roughness, optical throughput) for each single component and more complex and expensive alignment of the complete system.
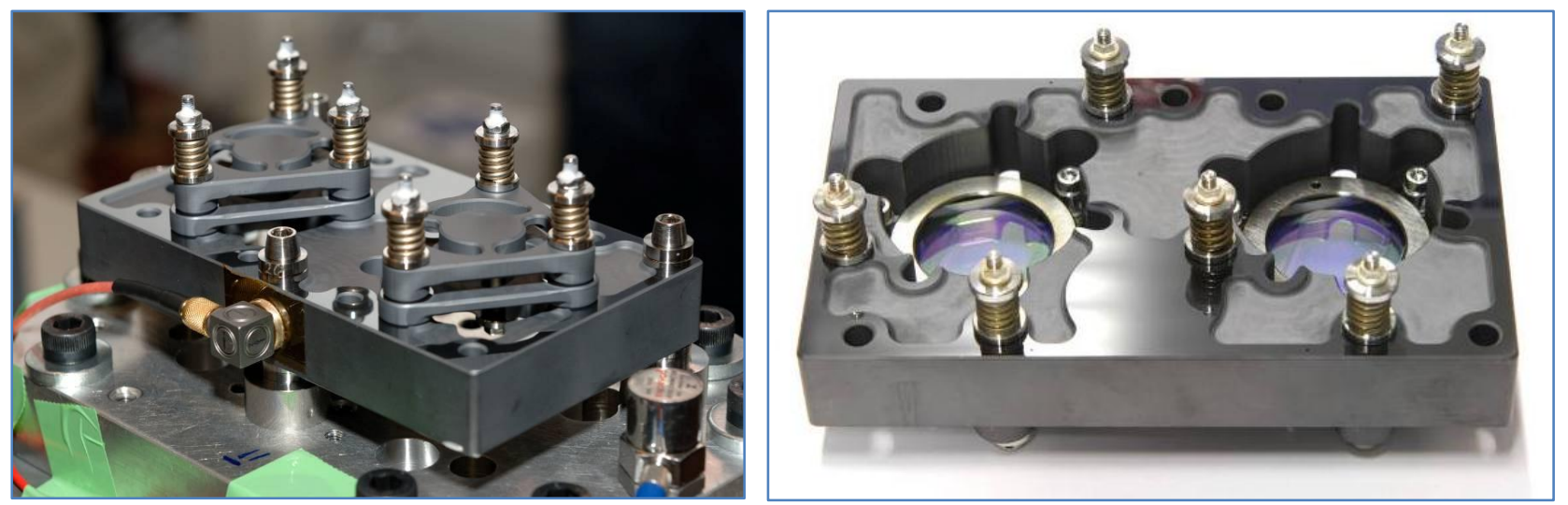

Figure 5: Test specimen for component level testing of stable mounting of SSiC mirrors (left) and Fused Silica beam splitters (right). Credits: TNO/Fred Kamphues 
Ultra-stable mounting of optical components is required to achieve the severe beam pointing, beam tilt, OPD and WFE requirements shown in Table 2, both after vibration loads and over a wide temperature range of more than 190K. The worst case number of optical surfaces that are passed by one of the BAM beams amounts up to 18, each surface adding to the total beam tilt and wave front error.

Reflection of the beams is accomplished by flat SSiC mirrors, polished down to $<2 \mathrm{~nm}$ rms WFE, coated with protective Silver and mechanically spring-mounted to monolithic brackets on the SSiC baseplate. Due to the SSiC-SSiC interface with the BAM structure these mirrors are less sensitive to temperature changes and the required tilt stability of less than 2 micro-rad is mainly influenced by mechanical vibration and shock loads during launch and PLM bi-pod release.

For the transmissive beam splitters, made of Fused Silica and having the same tilt stability requirement of $2 \mathrm{micro-rad,}$ another design approach needed to be developed as the glass-SSiC interface is not only susceptible to mechanical loads but also to temperature changes. In the final design solution, a thermal compensation mechanism using PEEK and no adhesive interface is used.

For both the SSiC mirror and Fused Silica beam splitter mounting configurations, extensive technology development test campaigns at component level have been performed in the early design phase to arrive at the final design solution (see Figure 5). With these component test campaigns, including several design iterations, finally tilt stabilities in the required range of 2 micro-rad were demonstrated.
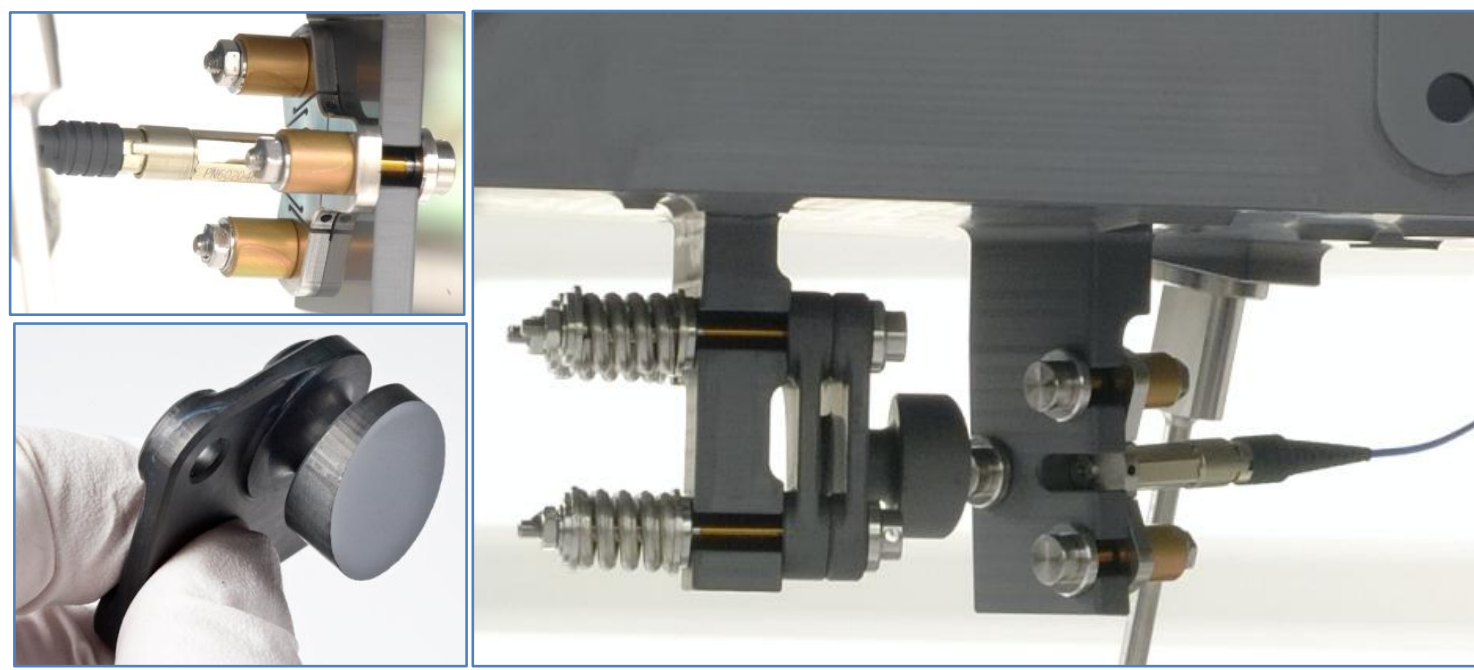

Figure 6: The cryogenic fiber collimator (right; Credits: TNO/Fred Kamphues) with the two dominating parts: fiber connector interface to SSiC BAM structure (upper left; Credits: TNO/Fred Kamphues) and the strongly curved off-axis parabolic SSiC mirror (lower left; Credits: TNO/Leo Ploeg).

Another significant technology development program was allocated to the fibre collimator because at start of the Gaia BAM program, cryogenic fibre collimators were not available at all.

With the main challenge to stay within the budgeted requirements of WFE and beam pointing stability allocated to the fibre collimator, both under mechanical vibration loads and a wide thermal range of $190 \mathrm{~K}$, the development was dominated by two components:

- $\quad$ The stable interface between the customer supplied laser source, a space qualified polarization maintaining fibre with standard AVIM connector, and the SSiC bar structure of the BAM system. The development is mainly driven by the cool-down from ambient to $100 \mathrm{~K}(\Delta \mathrm{T}$ of $190 \mathrm{~K})$ that needs to be taken into account for the different coefficients of thermal expansion of materials applied ( $\mathrm{ZrO} 2$ fibre ferrule, Titanium fibre tip, Steel connector body, SSiC BAM structure); in the final design configuration stabilities of less than $750 \mathrm{~nm}$ axial and $250 \mathrm{~nm}$ radial were achieved (where $<2 \mu \mathrm{m}$ axial and $<1 \mu \mathrm{m}$ radial were required). 
- A strongly curved off-axis parabolic SSiC mirror to make a perfect collimated beam from the diverging light emitted by the fibre light source. The main difficulty of this collimator mirror, beside that it is made of silicon carbide, is it small radius of curvature $(\mathrm{R}=50.17 \mathrm{~mm})$ over an effective aperture of only $10 \mathrm{~mm}$, maintaining a surface shape error of $\leq 12.5 \mathrm{~nm} \mathrm{rms}$ and a surface roughness of $\mathrm{Rq} \leq 6 \mathrm{~nm}$. With this unique combination of requirements, worldwide no suppliers could be found that could guarantee the delivery of such a state-of-the-art component in time. In close cooperation between TNO and the Leibniz Institute of Surface Modification (IOM), a manufacturing process of iterative robot machine polishing and Plasma Jet Machining was developed, which resulted in the realization of flight mirrors with a surface error in the range of $4.4-7.2 \mathrm{~nm} \mathrm{rms}$ and surface roughness better than $6 \mathrm{~nm}$ rms.

Other constraints that are involved with the design and implementation of the SSiC opto-mechanical BAM system are the stiffness of SSiC-SSiC interfaces (lower allowance tolerances for spread in pre-loads), the required manufacturing flatness of SSiC interface surfaces (fracture sensitivity), the behaviour of other materials in contact with SSiC, the friction behaviour specific to SSiC surfaces and the impact of the porosity of SSiC, if not CVD coated, on optical properties (reflection and stray light of SSiC mirrors).

\section{GAIA BAM REALIZATION AND INTEGRATION}

Based on the design definition of TNO, manufacturing of the SSiC parts for Gaia BAM, as for all Gaia SSiC part, has been performed by Boostec (nowadays Mersen) under control of Astrium. The most complex parts to be manufactured were the periscopes and the monolithic 1 meter length baseplates with all brackets for optical components included and the open backside structure (see Figure 4 right). For the design of the baseplates the main constraint was with the location and direction of mirror and beam splitter brackets such that all contact surfaces can be grinded to achieve the minimum needed flatness $(5 \mu \mathrm{m})$. Sufficient flat and reflective reference surfaces for alignment purposes were polished by TNO directly on the baseplates after delivery.
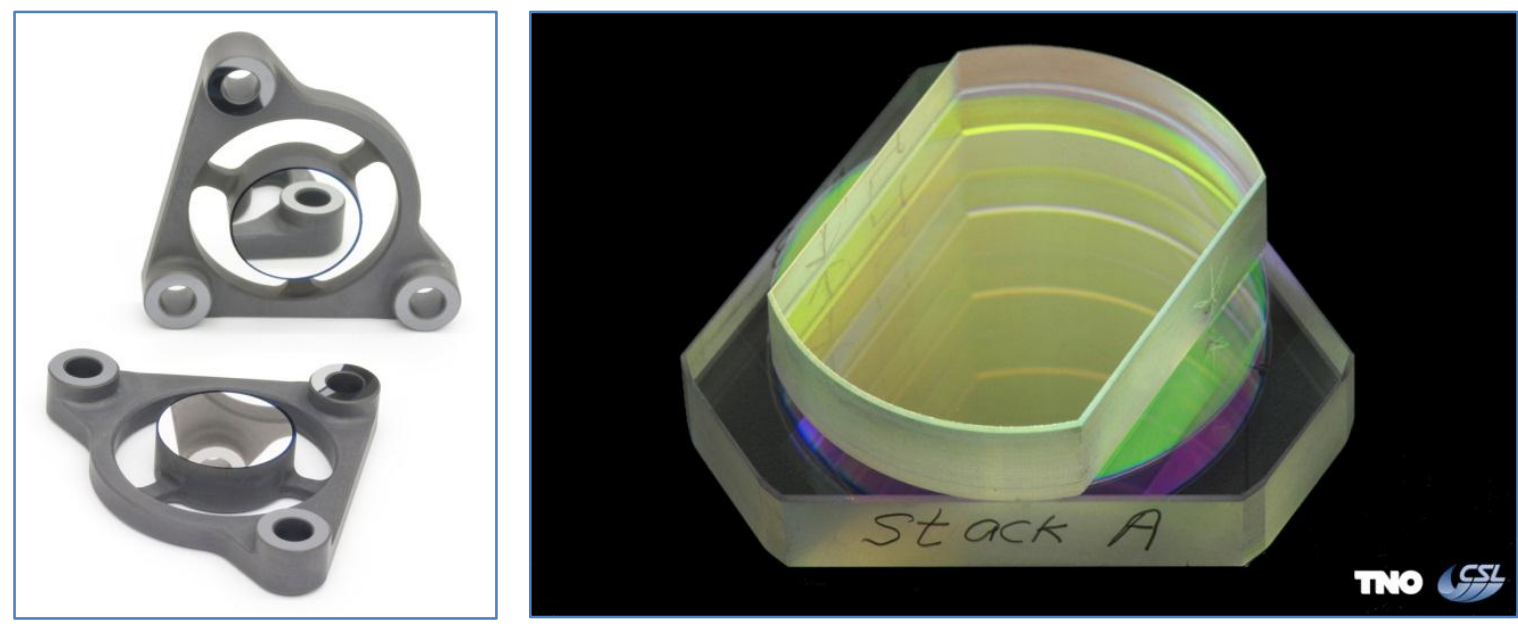

Figure 7: Polished and coated SSiC flat folding mirrors (left; Credits: TNO/Leo Ploeg) and optical contacted fused silica beam splitter (right; Credits: TNO/Fred Kamphues)

The SSiC mirrors were delivered as semi-finished blanks; polishing to high-quality optical components was developed and completed by TNO, using conventional diamond paste polishing for the flat folding mirrors (Figure 7 left) and the iterative process of robot polishing and Plasma Jet Machining of IOM for the off-axis parabolic collimator mirrors. Tuning of the thickness and tilt angle of the alignment shims is executed as part of the alignment process.

The glass-optics in the BAM system were manufactured by TNO; for the beam splitters (Figure 7 right), optical contacting of Fused Silica plates with a beam splitter coating in between is applied.

All optical coatings were designed, qualified and manufactured by Centre Spatial de Liège (CSL): protected Silver on SSiC mirrors, filter coating on beam splitters and AR coatings on beam splitters and polarizers. 
Integration of all parts, SSIC and non-SSiC, to a working BAM system has been a complex and delicate task involving specially developed tools and operations by specially trained workforces. Apart from working in ISO class 6 cleanroom environments and applying all rules and procedures custom for building space hardware, the specifically fragile properties of SSiC dictate the pace and sequence of steps. The amount of pre-load applied on bolted interfaces is one of the issues of concern; due to the mechanical surface properties of SSiC and to maintain the required alignment stability of the BAM, the allowable spread in pre-load is limited to prevent gapping on one hand and fracture due to highs stresses on the other hand. For each unique fastener interface therefore the torque-preload ratio needed to be calibrated.
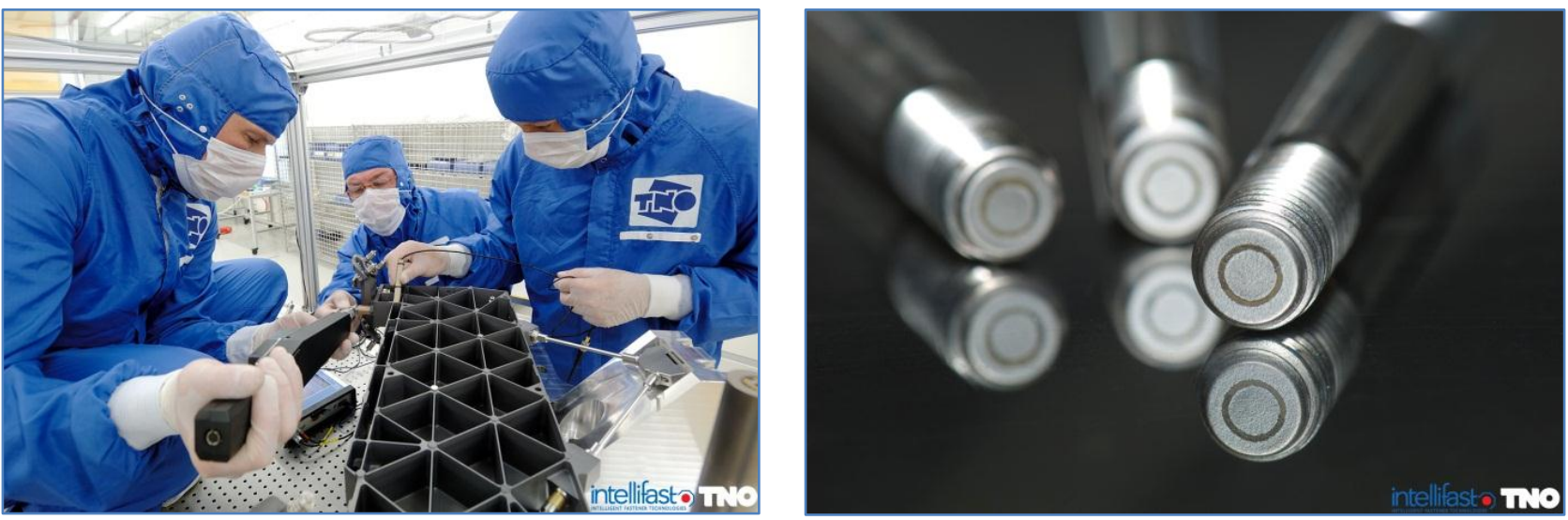

Figure 8: Gaia BAM periscope bolt installation with PMTS (left; Credits: TNO/Fred Kamphues) and Titanium fasteners equipped with PMTS structure (right; Credits: TNO/Fred Kamphues)

For the large M8 fasteners, used to mount the SSiC periscopes to the SSiC baseplates with high pre-load, even this calibration was not considered safe enough. To enable in-situ preload measurement, special ultrasonic transducers have been used, the so-called Permanent Mounted Transducer System (PMTS) developed by Intellifast GmbH (see Figure 8). With these transducers a repeatable accuracy of better than $+/-3 \%$ pre-load, independent of the operator skill and including thermal cycling for qualification, has been demonstrated by TNO before application. For use in Gaia BAM, the Titanium M8 fasteners were equipped with a special high temperature coating instead of the standard transducer tin electrode material, in order to avoid the whisker growth in space.
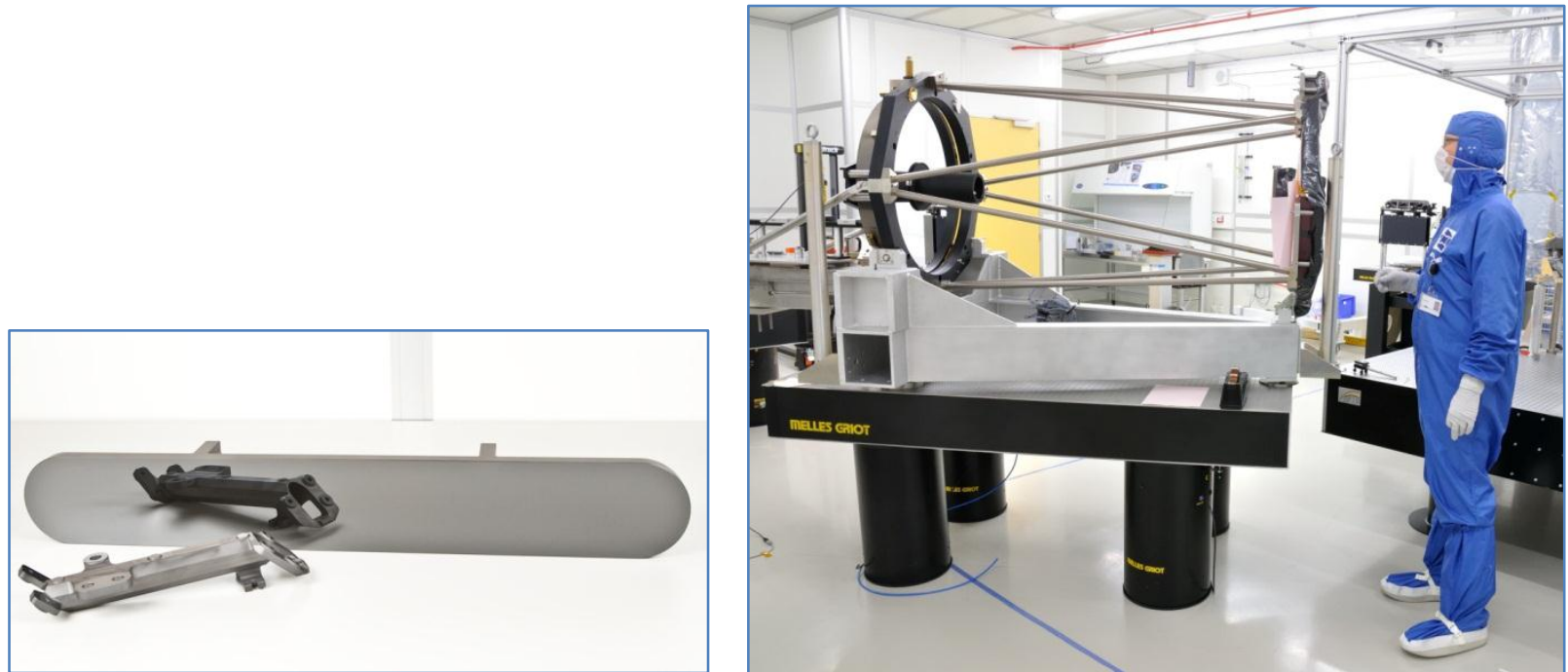

Figure 9: SSiC reference flat with periscope mirrored (left; Credits: TNO/Leo Ploeg) and the Astrium COL70 collimator used for final OPD verification (right; Credits: TNO/Fred Kamphues) 
Following positioning and alignment of the BAM bars with the Iso-static Mounts to the defined interface with the Gaia PLM torus, by far the major task during integration of the BAM system was allocated to optical fine alignment of the collimators and flat folding mirrors by tuning of shims. Methods, tools and procedures for measurement and alignment to the required values have been developed making optimum use of the current state-of-the-art accuracies for optical test equipment and dedicated set-ups. A special SSiC flat reference mirror with very accurate polished reference surfaces (specified for surface form, flatness and parallelism) at the base length difference of $540 \mathrm{~mm}$ was procured to facilitate accurate alignment (Figure 9 left). Especially in the final stage, to arrive at the required low values for beam pointing, differential beam tilt and OPD (Table 2) for each of the beam sets A-A' and C-C', new techniques, procedures and tooling for iterative polishing and measurement of alignment shims needed to be developed. Various measurement methods involving interferometry and the TNO Nanomefos system were applied. In the final stage of the program, a large $700 \mathrm{~mm}$ collimator (the COL70), kindly provided on loan by EADS Astrium, was used to arrive at the required accuracy level for final OPD verification (Figure 9 right).

\section{GAIA BAM VERIFICATION \& QUALIFICATION TESTING}

After completion of integration and alignment and prior to environmental qualification testing, the performance of the BAM system has been verified by a set of measurements at ambient conditions. The alignment parameters such as baseline angle, base length difference, beam pointing, differential beam tilt and OPD were already included in the final alignment verification. Additionally the WFE, transmission and polarization (extinction ratio) were determined. All requirements were fulfilled except for transmission of beam set C-C', the channel with the most optical surfaces in the light path.

Next step in the verification program was to demonstrate, through an environmental qualification test campaign, that the BAM system survives and still meets all performance requirements after exposure to vibration (launch) and after transient to low temperature (L2 conditions).

Vibration testing of the BAM system was performed at an external facility of CSL Belgium (see Figure 10); both BAM bars 1 and 2 were individually subjected to pre-defined sine and random vibration spectra derived from Soyuz launch loads. Before testing the BAM PFM, a mechanically representative structural model of BAM bar 1 was tested to demonstrate that models and prediction were within acceptable range, to exclude any risk of damage during the flight model testing. Testing is performed in each of the three perpendicular axes $\mathrm{X}, \mathrm{Y}$ and $\mathrm{Z}$; notching of the input spectra, where needed, was agreed prior to test and iteratively tuned with customer Astrium along the test. Following the vibration test campaign, the key performance parameters (pointing and beam tilt alignment stability and OPD) were measured again at TNO to demonstrate that the alignment did not change more than the predicted settling effects and that the performance requirements were still met.
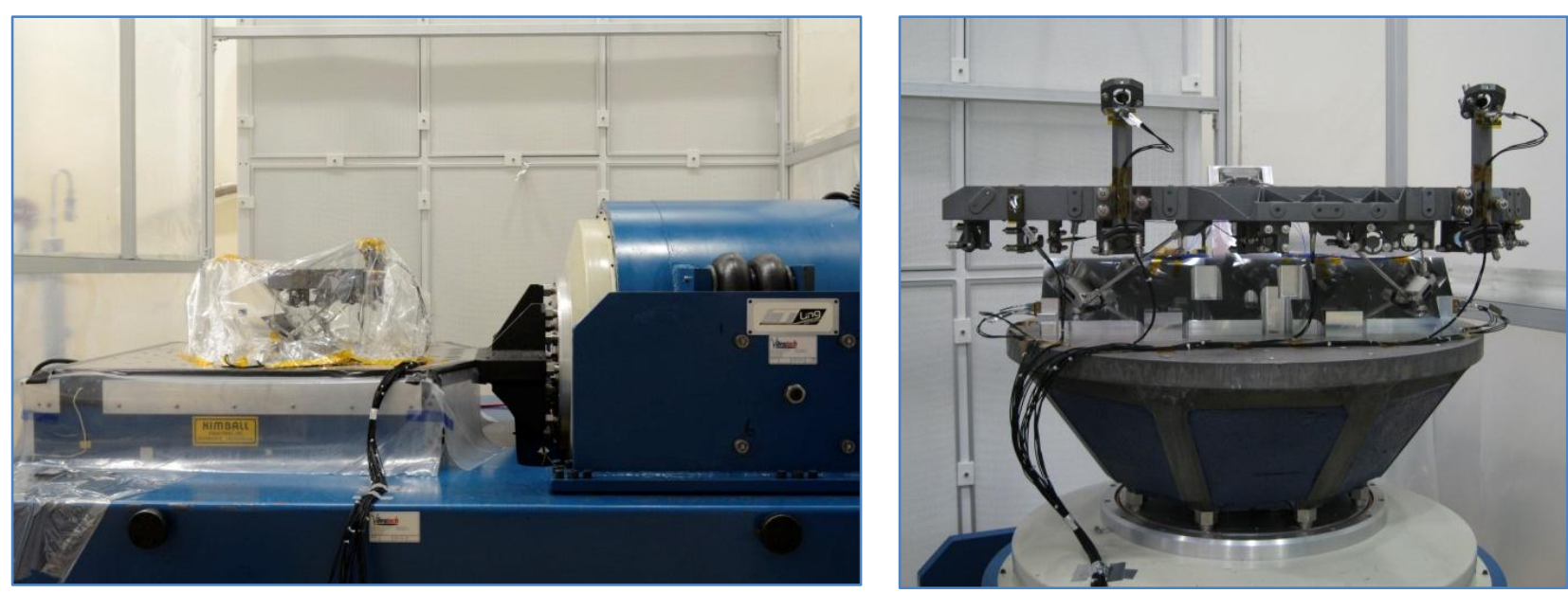

Figure 10: Vibration testing of BAM PFM at CSL. Bar1 Z-axis vibration on slip table (left; Credits: TNO/Fred Kamphues) and bar2 $\mathrm{X}$-axis vibration on head expander (cleanliness protective foil temporarily removed) (right; Credits: TNO/Jeroen Mekking) 
Thermal cycling of the BAM PFM bars was performed in the VCF Thermal Vacuum chamber of TNO (Figure 11, right); 4 thermal cycles, covering a temperature range from $323 \mathrm{~K}$ to $100 \mathrm{~K}$, have been applied of which 1 cycle down to the minimum qualification temperature of $93 \mathrm{~K}$. Performance measurements of the BAM system at operational conditions were accomplished at a temperature of $110 \mathrm{~K}$.

A dedicated thermal-mechanical balanced set-up of Invar (Figure 11, left) was used to achieve the necessary stability for the interface between the test chamber and the SSiC BAM structure and to secure the required stability for accurate alignment performance verification. Despite this set-up and all other precautions, difficulties were encountered in the stability of measuring the beam pointing performance which could be solved after some modification and additional stand-alone testing of the set-up. The beam pointing values shown in Table 3 with verified BAM system performance results present the worst case situation at $110 \mathrm{~K}$ including the linearly added uncertainties caused by the experienced testing constraint.
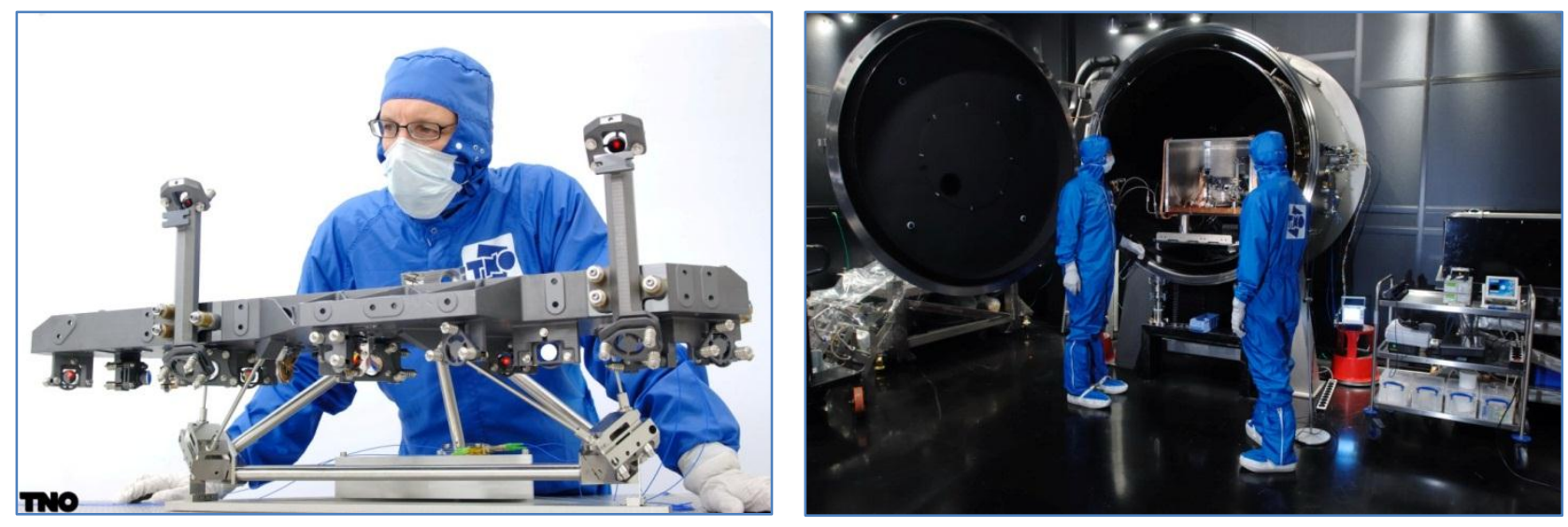

Figure 11: Thermal testing of BAM PFM: bar 2 on Invar set-up (left) and BAM in TNO VCF Thermal Vacuum chamber (right). Credits: TNO/Fred Kamphues

The final BAM system performance results achieved at the completion of the entire verification and space qualification testing campaign are presented in Table 3. The values shown include the performance at operational temperature (measured at $110 \mathrm{~K}$ ) and shows the worst case combination of individual test data and measurement uncertainties. For beam pointing and transmission of beam set C-C', the non-compliances have been waivered at Gaia system level.

Table 3: BAM system performance after completion of verification and qualification testing (worst case values). The underlined results are not compliant to the requirement but accepted at system level by waiver.

\begin{tabular}{|l|l|l|}
\hline Requirement: & Measured: & Required: \\
\hline Baseline angle: & $106,5^{\circ} \pm 23 \mu \mathrm{rad}$ & $106,5^{\circ} \pm 50 \mu \mathrm{rad}$ \\
\hline Base length difference: & $\begin{array}{l}\text { A-A': } 540.0 \mathrm{~mm} \\
\text { C-C': } 540.7 \mathrm{~mm}\end{array}$ & $540-660 \mathrm{~mm}( \pm 1 \mathrm{~mm})$ \\
\hline Beam pointing: & $\begin{array}{l}\text { A-A': } 120 \mu \mathrm{rad} \\
\text { C-C' } \underline{110} \mu \mathrm{rad}\end{array}$ & $<100 \mu \mathrm{rad}$ \\
\hline Differential beam tilt: & $\begin{array}{l}\text { A-A': } 38 \mu \mathrm{rad} \\
\text { C-C': } 42 \mu \mathrm{rad}\end{array}$ & $<50 \mu \mathrm{rad}$ \\
\hline OPD: & $\begin{array}{l}\text { A-A': } 8.2 \mu \mathrm{m} \\
\text { C-C': } 4.4 \mu \mathrm{m}\end{array}$ & $<8.5 \mu \mathrm{m}$ \\
\hline WFE: & $\begin{array}{l}\text { Beam set A: } 24 \mathrm{~nm} \mathrm{rms} \\
\text { Beam set C: } 20 \mathrm{~nm} \mathrm{rms}\end{array}$ & $25 \mathrm{~nm} \mathrm{rms}$ \\
\hline Transmission: & $\begin{array}{l}\text { Beam set A: } 0.182-0.215 \\
\text { Beam set C: } \underline{0.073}-\underline{0.132}\end{array}$ & $>0.15$ \\
\hline
\end{tabular}




\section{REFERENCES}

[1] ESA Gaia mission website: http://www.esa.int/esaSC/120377_index_0_m.html

[2] EADS Astrium Gaia mission website: http://www.astrium.eads.net/en/programme/gaia.html

[3] TNO Gaia BAM website:

http://www.tno.nl/content.cfm?context=thema\&content=prop_case\&laag1=897\&laag2=921\&laag3=116\&item $\underline{\mathrm{id}=585 \& \mathrm{Taal}=2}$

[4] E.A. Meijer et al. "Picometer metrology for the GAIA Mission". Proc. SPIE 7439, 743915 (2009). 